\title{
RIEMANNIAN MEANS AS SOLUTIONS OF VARIATIONAL PROBLEMS
}

\author{
LUÍS MACHADO, F. SILVA LEITE AND KNUT HÜPER
}

\begin{abstract}
We formulate a variational problem on a Riemannian manifold $M$ whose solutions are piecewise smooth geodesics that best fit a given data set of time labelled points in $M$. By a limiting process, these solutions converge to a single point in $M$, which we prove to be the Riemannian mean of the given points for some particular Riemannian manifolds such as Euclidean spaces, connected and compact Lie groups, and spheres.
\end{abstract}

\section{Introduction}

One way of defining the arithmetic mean of a finite set of points, $q_{0}, \ldots, q_{N}$, in the Euclidean space $\mathbb{R}^{n}$ is as the unique point that minimizes the sum of the squared distances to the given points $q_{i}$; that is,

$$
\frac{1}{N+1} \sum_{i=0}^{N} q_{i}=\min _{q \in \mathbb{R}^{n}} \sum_{i=0}^{N} d^{2}\left(q, q_{i}\right),
$$

where $d(q, p)=\|q-p\|$ is the usual Euclidean distance in $\mathbb{R}^{n}$.

Although there are a wide number of distinct 'means' in Euclidean spaces, the one defined above is the most commonly used to average elements on linear spaces and, depending on the application, it is also known as the centroid (or center of mass), average or barycenter.

When generalizing the above optimization problem to general Riemannian manifolds, several notions of mean may arise, depending on the choice of the distance function $[13,14]$.

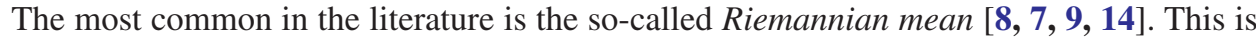
defined as comprising those points that minimize the sum of the squared geodesic distances to the given points $q_{i}$. Contrary to the Euclidean case, we have no guarantee that solutions of this optimization problem are unique. Indeed, when the sample points are symmetrically distributed in a symmetric space, the Riemannian mean is not a singleton [9].

This concept of a geometric mean has been extensively studied by several authors. We refer to Buss and Fillmore [2], Galperin [4], Moakher [13, 14], Krakowski [9], and ongoing work by Hüper and Manton, to mention a few.

In [2], the authors used the notion of spherical averages to develop a method to generate interpolating splines in the unit $n$-sphere $S^{n}$. Apart from the spheres, the Riemannian mean on the connected and compact Lie group of the special orthogonal matrices $\mathrm{SO}(n)$ has received much attention in the past few years $[13,14]$. This is due to the fact that in many applications of fuzzy control, vision, robotics, biomechanics and geophysics, among others, most of the experimental data are given as a sequence of rotation matrices. For interest, we 
refer to [1], where a very interesting geometric model of the human spine, based in threedimensional rotations, is described.

Motivated by the study of fitting smoothing cubic splines to data on Riemannian manifolds [11], originally studied in the two-dimensional sphere $S^{2}$ by Jupp and Kent [6], in the present paper we formulate a variational problem on a Riemannian manifold $M$ that is related to the Riemannian mean defined above in a sense that will be made precise below.

This paper is organized as follows. In Section 2, we gather all the necessary information about Riemannian means that will be needed in the paper. In Section 3, we consider the following variational problem on the Riemannian manifold $M$ : "Given a set of points in $M, q_{0}, \ldots, q_{N}$, and a partition of the unit time interval $[0,1], 0=t_{0}<\ldots<t_{N}=1$, find a curve $\gamma$ in $M$ that minimizes

$$
J(\gamma)=\frac{1}{2} \sum_{i=0}^{N} d^{2}\left(q_{i}, \gamma\left(t_{i}\right)\right)+\frac{\lambda}{2} \int_{0}^{1}\left\langle\frac{d \gamma}{d t}, \frac{d \gamma}{d t}\right\rangle d t,
$$

over the class of all piecewise smooth paths $\gamma:[0,1] \rightarrow M$, where $d$ is the geodesic distance in $M$ and $\lambda(>0)$ is a smoothing parameter."

Solutions of this variational problem are shown to be piecewise smooth geodesics that best fit the given data (points and instants of time). When the smoothing parameter $\lambda$ is converging to $+\infty$, the Euler-Lagrange equations associated to this variational problem converge to a single point in $M$. Finally, we prove that when explicit forms for geodesics on Riemannian manifolds are available, this single point is nothing other than the Riemannian mean of the points $q_{0}, \ldots, q_{N}$. Such is the case for Euclidean spaces, connected and compact Lie groups, and spheres.

\section{Riemannian means}

In Euclidean spaces, the center of mass of a set of points $q_{0}, \ldots, q_{N}$, having attached to each $q_{i}$ the weight $\omega_{i}(\geqslant 0)$, is

$$
q_{E}=\frac{\sum_{i=0}^{N} \omega_{i} q_{i}}{\sum_{i=0}^{N} \omega_{i}} .
$$

When all the weights $\omega_{i}$ are equal, we obtain the arithmetic mean of the points $q_{0}, \ldots, q_{N}$.

But, since the latter can be interpreted as the unique solution of the following optimization problem

$$
\min _{q \in \mathbb{R}^{n}} \sum_{i=0}^{N} d^{2}\left(q_{i}, q\right),
$$

where $d$ denotes Euclidean distance, we can generalize this concept to Riemannian manifolds, and define the so-called Riemannian mean (see, for instance, $[7,9,13,14]$ ).

To this end, let us consider a connected and complete Riemannian manifold $M$, and denote by $\langle\cdot, \cdot\rangle$ its Riemannian metric. If $t \longmapsto \gamma(t)$ is a smooth curve on $M$ and $t \longmapsto Y(t)$ is a smooth vector field along $\gamma$, we denote by $D Y / d t$ the covariant derivative of $Y$ along $\gamma$ with respect to the Riemannian connection (Levi-Civita connection) $\nabla$. Let $\exp _{p}: T_{p} M \rightarrow M$ denote the geodesic map, as defined by Milnor [12].

Now, given a set of points in $M, q_{0}, \ldots, q_{N}$, let us consider the counterpart of the 
optimization problem $\left(\mathscr{P}_{1}\right)$ to the Riemannian manifold $M$,

$$
\min _{q \in M} \sum_{i=0}^{N} d^{2}\left(q_{i}, q\right),
$$

where $d$ stands for the Riemannian distance (or geodesic distance) on $M$, and is defined for points $p$ and $q$ sufficiently close as

since

$$
d^{2}(p, q)=\left\langle\exp _{p}^{-1}(q), \exp _{p}^{-1}(q)\right\rangle
$$

$$
\begin{aligned}
\gamma:[0,1] & \longrightarrow M, \\
s & \longmapsto \gamma(s)=\exp _{p}\left(s \exp _{p}^{-1}(q)\right),
\end{aligned}
$$

explicitly parameterizes the shortest geodesic arc joining $p$ to $q$ (see [7]).

Contrary to the Euclidean situation, there is no a unique solution for problem $\left(\mathcal{P}_{2}\right)$, so we adopt the following definition for the Riemannian mean, given by Krakowski [9].

Definition 2.1. Let $Q=\left\{q_{0}, \ldots, q_{N}\right\}$ be a finite set of points in $M$, and consider the following function defined on $M$ :

$$
\begin{aligned}
\Phi_{Q}: M & \longrightarrow \mathbb{R}, \\
q & \longmapsto \Phi_{Q}(q)=\sum_{i=0}^{N} d^{2}\left(q_{i}, q\right) .
\end{aligned}
$$

The Riemannian mean $\bar{Q} \subset M$ is the set of points at which $\Phi_{Q}$ attains its global minimum.

In the case when the Riemannian mean is a singleton, we call it the Riemannian mean of the points $q_{0}, \ldots, q_{N}$.

A necessary condition for $q \in M$ to be in the Riemannian mean of the set of points $Q$ may be found in [9], and is stated in the following theorem.

THEOREM 2.1. Suppose that there exists a normal neighborhood $\mathcal{U}$ of $q \in M$ such that $Q \subset \mathcal{U}$. Then $q$ is a critical point for $\Phi_{Q}$ if and only if

$$
\sum_{i=0}^{N} \exp _{q}^{-1}\left(q_{i}\right)=0 .
$$

In this work, we are particularly interested in considering the Riemannian mean for special Riemannian manifolds such as connected and compact Lie groups, and spheres.

Before stating the counterparts of the above theorem for these two cases, we need to introduce some terminology that will be of particular interest in the derivation of some of the results presented here.

Let $G$ be a connected and compact Lie group, and let us denote by $\mathfrak{g}$ its Lie algebra. It can be proved (see, for instance, the work of Helgason [5] or Milnor [12]) that we can endow $G$ with a bi-invariant Riemannian metric. We retain the notation $\langle\cdot, \cdot\rangle$ to denote the bi-invariant Riemannian metric in $G$.

Geodesics in $G$ are the one-parameter subgroups of $G$ or their translates; therefore, if $x$ and $y$ are points sufficiently close in $G$, we can explicitly parameterize the geodesic joining them as

$$
\begin{aligned}
\gamma:[0,1] & \longrightarrow G, \\
t & \longmapsto \gamma(t)=x \exp (t Z),
\end{aligned}
$$

where $\exp Z=x^{-1} y$. 
So, following Munthe-Kaas et al. [15], we can define a bi-invariant distance function in $G$, if we define the distance between $x$ and $y$ as being the length of this minimizing geodesic; that is,

$$
\begin{aligned}
d(x, y) & =\int_{0}^{1}\left\langle\dot{\gamma}(t),\left.\dot{\gamma}(t)\right|_{T_{\gamma(t)} G} ^{1 / 2} d t\right. \\
& =\int_{0}^{1}\langle\gamma(t) Z, \gamma(t) Z\rangle_{T_{\gamma(t)} G}^{1 / 2} d t \\
& =\int_{0}^{1}\langle Z, Z\rangle^{1 / 2} d t \\
& =\langle Z, Z\rangle^{1 / 2} .
\end{aligned}
$$

In order to guarantee the invertibility of the exponential mapping in $G$, in what follows we will restrict the set of points in $G, q_{0}, \ldots, q_{N}$, to lie in the image of the domain of injectivity of the exponential mapping that we denote by $\mathscr{B}$ (Lazard and Tits [10]). In this case, the inverse of the exponential will be called the logarithm and denoted by 'log'.

Therefore, attending to the above considerations, we can explicitly parameterize the minimizing geodesic in $G$ joining two points $q$ and $q_{i}$, in $\exp \mathcal{B}$, as

$$
\begin{aligned}
\gamma:[0,1] & \longrightarrow G, \\
t & \longmapsto \gamma(t)=q \exp \left(t \log \left(q^{-1} q_{i}\right)\right) .
\end{aligned}
$$

This means that the velocity vector (at $t=0$ ) of the minimizing geodesic joining $q$ to $q_{i}$ is given by

$$
\frac{d \gamma}{d t}(0)=q \log \left(q^{-1} q_{i}\right)
$$

which, according to (2.1), is equivalent to $\exp _{q}^{-1}\left(q_{i}\right)=q \log \left(q^{-1} q_{i}\right)$.

This can now be used to characterize the Riemannian mean in the connected and compact Lie group $G$.

Proposition 2.1. $q \in G$ is a critical point for the function $\Phi_{Q}$, defined in $G$ by

$$
\Phi_{Q}(q)=\sum_{i=0}^{N}\left\langle\log \left(q^{-1} q_{i}\right), \log \left(q^{-1} q_{i}\right)\right\rangle
$$

if and only if

$$
\sum_{i=0}^{N} \log \left(q^{-1} q_{i}\right)=0
$$

Numerical methods concerning the computation of the Riemannian mean on the special orthogonal Lie group $\mathrm{SO}(n)$ can be found in Krakowski [9] and in ongoing work by Manton and Hüper. The former is based on the gradient steepest descent method, while the latter is based on Newton's method. We will not go into further details concerning this matter, since it would divert us away from the objectives of this paper.

Now, if we consider the $n$-dimensional unit sphere,

$$
S^{n}=\left\{y \in \mathbb{R}^{n+1}:\langle y, y\rangle=1\right\},
$$


endowed with the Riemannian metric induced by the Euclidean inner product

$$
\langle x, y\rangle=x^{\top} y, \quad x, y \in \mathbb{R}^{n+1},
$$

then geodesics in $S^{n}$ are the great arc circles that can be given explicitly as

$$
\begin{aligned}
\gamma: \mathbb{R} & \longrightarrow S^{n} \\
t & \longmapsto \gamma(t)=q \cos (\|v\| t)+\frac{v}{\|v\|} \sin (\|v\| t),
\end{aligned}
$$

where $q \in S^{n}$ and $v \in T_{q} S^{n}$.

Similarly to what has been done for the Lie group case, we will define the spherical distance between two non-antipodal points $q$ and $q_{i}$ as being the length of the shortest minimizing geodesic joining them. However, since this geodesic can be parameterized explicitly by

$$
\begin{aligned}
\gamma:[0,1] & \longrightarrow S^{n}, \\
t & \longmapsto \gamma(t)=q \cos (t \alpha)+\left(q_{i}-q \cos \alpha\right) \frac{\sin (t \alpha)}{\sin \alpha},
\end{aligned}
$$

where $\left.\alpha=\cos ^{-1}\left\langle q, q_{i}\right\rangle \in\right] 0, \pi$ [, we deduce, as expected, that $d\left(q, q_{i}\right)=\alpha$.

Now, the velocity vector of the shortest geodesic joining $q$ to $q_{i}$ is the vector

$$
\frac{d \gamma}{d t}(0)=\frac{\alpha}{\sin \alpha}\left(q_{i}-q \cos \alpha\right),
$$

and we have the following characterization for the Riemannian mean in $S^{n}$.

Proposition 2.2. A point $q \in S^{n}$ is a critical point for $\Phi_{Q}$, defined in $S^{n}$ by

$$
\Phi_{Q}(q)=\sum_{i=0}^{N} \cos ^{-2}\left\langle q, q_{i}\right\rangle
$$

where $\cos ^{-1}$ stands for the inverse function of the cosine, if and only if

$$
\sum_{i=0}^{N} \frac{\cos ^{-1}\left\langle q, q_{i}\right\rangle}{\sqrt{1-\left\langle q, q_{i}\right\rangle^{2}}}\left(q_{i}-q\left\langle q, q_{i}\right\rangle\right)=0 .
$$

REMARK 2.1. A different proof of the above theorem can be found in Buss and Fillmore [2], where they develop a method for computing interpolating splines based on weighted spherical averages. They also present numerical methods for computing the spherical mean.

\section{Formulation of a variational problem}

In this section we formulate a variational problem on $M$, whose solutions are piecewise smooth geodesics fitting a given data set of points at given instants of time. In this work, we use the terms 'smooth' and 'differentiable' interchangeably to denote $C^{\infty}$ maps. Before formulating our variational problem, we define the family of admissible paths.

Let us start with a collection of points in $M$,

$$
q_{0}, q_{1}, \ldots, q_{N},
$$

and a partition of the unit interval $[0,1]$,

$$
0=t_{0}<t_{1}<\ldots<t_{N}=1 .
$$


Definition 3.1. By an admissible path will be meant a continuous $\left(C^{0}\right)$ and piecewise smooth path, $\gamma:[0,1] \rightarrow M$, satisfying the following conditions.

- $\left.\gamma\right|_{\left[t_{i}, t_{i+1}\right]}$ is smooth, for $i=0, \ldots, N-1$.

- The left and right covariant derivatives at the points $t_{i}$ exist as follows:

$$
\lim _{t \rightarrow t_{i}^{-}} \frac{d \gamma}{d t}(t)=\frac{d \gamma}{d t}\left(t_{i}^{-}\right), \quad \lim _{t \rightarrow t_{i}^{+}} \frac{d \gamma}{d t}(t)=\frac{d \gamma}{d t}\left(t_{i}^{+}\right) .
$$

$\Omega$ will denote the set of all admissible paths on $M$.

Our main objective in this section is to find an admissible path on $M$ that best fits the given points (3.1) at the given instants of time (3.2), in the sense that the functional

$$
J(\gamma)=\frac{1}{2} \sum_{i=0}^{N} d^{2}\left(q_{i}, \gamma\left(t_{i}\right)\right)+\frac{\lambda}{2} \int_{0}^{1}\left\langle\frac{d \gamma}{d t}, \frac{d \gamma}{d t}\right\rangle d t,
$$

defined over $\Omega$, should take the smallest possible value.

Here $d(p, q)$ denotes the Riemannian distance between $p$ and $q$, and $\lambda \in \mathbb{R}^{+}$plays the role of a smoothing parameter.

In order to find the critical paths for $J$, one needs to define an admissible variation of $\gamma \in \Omega$.

Definition 3.2. Let $\gamma:[0,1] \rightarrow M$ be an admissible path in $M$, in the sense of Definition 3.1. By a one-parameter variation of $\gamma$ will be meant a function

$$
\alpha:(-\varepsilon, \varepsilon) \times[0,1] \rightarrow M,
$$

for some $\varepsilon>0$, such that:

- $\alpha(0, t)=\gamma(t)$

- $\alpha$ is smooth on each strip $(-\varepsilon, \varepsilon) \times\left[t_{i}, t_{i+1}\right], i=0, \ldots, N-1$.

Since for each $u \in(-\varepsilon, \varepsilon), \alpha_{u}$ is an admissible variation of $\gamma$, we may think of $\alpha$ as a 'smooth path' in $\Omega$, and its velocity vector $\partial \alpha / \partial u(0) \in T_{\gamma} \Omega$ can be interpreted as the vector field $W$ along $\gamma$ given by

$$
W(t)=\frac{\partial \alpha}{\partial u}(0, t) .
$$

Clearly, $W \in T_{\gamma} \Omega$ and we will refer to this vector field as the variational vector field associated with the variation $\alpha$.

We can think of $\Omega$ as an infinite-dimensional manifold and introduce the tangent space of $\Omega$ at a path $\gamma, T_{\gamma} \Omega$, as the set of all variational vector fields $t \longmapsto W(t)$ along $\gamma$ that are continuous and piecewise smooth on the domains $\left[t_{i}, t_{i+1}\right]$, for $i=0, \ldots, N-1$.

Hence, by exponentiating a vector field $W \in T_{\gamma} \Omega$, we obtain a one-parameter variation of $\gamma$, namely $\alpha:(-\varepsilon, \varepsilon) \times[0,1] \rightarrow M$, defined by

$$
\alpha(u, t)=\exp _{\gamma(t)}(u W(t)),
$$

for some $\varepsilon>0$. 
THEOREM 3.1. If $\alpha$ is a one-parameter variation of $\gamma \in \Omega$ and $W \in T_{\gamma} \Omega$ is the variational vector field associated to $\alpha$, then

$$
\begin{aligned}
& \left.\frac{d}{d u}\right|_{u=0} J\left(\alpha_{u}\right) \\
& \quad=-\sum_{i=0}^{N}\left\langle W\left(t_{i}\right), \lambda\left[\frac{d \gamma}{d t}\left(t_{i}^{+}\right)-\frac{d \gamma}{d t}\left(t_{i}^{-}\right)\right]+\exp _{\gamma\left(t_{i}\right)}^{-1}\left(q_{i}\right)\right\rangle+\lambda \int_{0}^{1}\left\langle\frac{D^{2} \gamma}{d t^{2}}, W\right\rangle d t,
\end{aligned}
$$

where we assume for brevity of notation that

$$
\frac{d \gamma}{d t}\left(0^{-}\right)=\frac{d \gamma}{d t}\left(1^{+}\right)=0 .
$$

Apart from adaptations to the present problem, the proof of Theorem 3.1 will follow closely what has been done in the literature for the variational theory of geodesics (see, for instance, Milnor [12] or do Carmo [3]).

Proof. Before we compute the value of $d / d u J\left(\alpha_{u}\right)$, we make some considerations analogous to what has been done in Krakowski [9].

Since $d\left(q_{i}, \gamma\left(t_{i}\right)\right)$ denotes the Riemannian distance between points $q_{i}$ and $\gamma\left(t_{i}\right)$, according to (2.1), when we consider the variation $\alpha$ of $\gamma$, defined by (3.4), one obtains the parameterized surface in $M$

$$
c_{i}(s, u)=\exp _{q_{i}}\left(s \exp _{q_{i}}^{-1}\left(\alpha_{u}\left(t_{i}\right)\right)\right), \quad s \in[0,1], u \in(-\varepsilon, \varepsilon) .
$$

Let us introduce the following vector fields associated to (3.7):

$$
c_{i}^{\prime}(s, u)=\frac{\partial}{\partial s} c_{i}(s, u), \quad \dot{c}_{i}(s, u)=\frac{\partial}{\partial u} c_{i}(s, u) .
$$

Since for fixed $u, s \longmapsto c_{i}(s, u)$ is a family of geodesics, $s \longmapsto \dot{c}_{i}(s, u)$ is a family of Jacobi vector fields along the family of geodesics $s \longmapsto c_{i}(s, u)$. This can now be used to derive the following:

$$
\begin{aligned}
& \frac{d}{d u} J\left(\alpha_{u}\right) \\
& \quad=\sum_{i=0}^{N} \int_{0}^{1}\left\langle\frac{D}{\partial u} c_{i}^{\prime}(s, u), c_{i}^{\prime}(s, u)\right\rangle d s+\lambda \int_{0}^{1}\left\langle\frac{\partial}{\partial u}\left(\frac{\partial \alpha}{\partial t}\right), \frac{\partial \alpha}{\partial t}\right\rangle d t \\
& =\sum_{i=0}^{N} \int_{0}^{1}\left\langle\frac{D}{\partial s} \dot{c}_{i}(s, u), c_{i}^{\prime}(s, u)\right\rangle d s+\lambda \int_{0}^{1}\left\langle\frac{\partial}{\partial t}\left(\frac{\partial \alpha}{\partial u}\right), \frac{\partial \alpha}{\partial t}\right\rangle d t \\
& =\sum_{i=0}^{N}\left\langle\dot{c}_{i}(1, u), c_{i}^{\prime}(1, u)\right\rangle+\lambda \int_{0}^{1} \frac{\partial}{\partial t}\left\langle\frac{\partial \alpha}{\partial u}, \frac{\partial \alpha}{\partial t}\right\rangle d t-\lambda \int_{0}^{1}\left\langle\frac{\partial \alpha}{\partial u}, \frac{D^{2} \alpha}{\partial t^{2}}\right\rangle d t .
\end{aligned}
$$

By considering $u=0$ in the above expression, we obtain

$$
\left.\frac{d}{d u}\right|_{u=0} J\left(\alpha_{u}\right)=-\sum_{i=0}^{N}\left\langle W\left(t_{i}\right), \exp _{\gamma\left(t_{i}\right)}^{-1}\left(q_{i}\right)+\left.\lambda \sum_{i=0}^{N-1}\left\langle W, \frac{d \gamma}{d t}\right\rangle\right|_{t_{i}^{+}} ^{t_{i+1}^{-}}-\lambda \int_{0}^{1}\left\langle W, \frac{D^{2} \gamma}{d t^{2}}\right\rangle d t .\right.
$$

and expression (3.5) follows immediately if we recall conditions (3.6). 
As a consequence of the previous theorem, we can characterize the critical (or stationary) points for the functional $J$ given by (3.3).

THEOREM 3.2. A necessary condition for $\gamma$ to be an extremal for the functional $J$ given by (3.3), over the class $\Omega$ of all piecewise smooth paths $\gamma$ on $M$ satisfying the conditions of Definition 3.1, is that for $t \in\left[t_{i}, t_{i+1}\right]$ and $i=0, \ldots, N-1, \gamma$ satisfies

$$
\frac{D^{2} \gamma}{d t^{2}}=0
$$

Moreover, at the knot points $t_{i}, \gamma$ satisfies the following regularity conditions:

$$
\frac{d^{j} \gamma}{d t^{j}}\left(t_{i}^{+}\right)-\frac{d^{j} \gamma}{d t^{j}}\left(t_{i}^{-}\right)= \begin{cases}0, & j=0(i=1, \ldots, N-1), \\ -\frac{1}{\lambda} \exp _{\gamma\left(t_{i}\right)}^{-1}\left(q_{i}\right), & j=1(i=0, \ldots, N) .\end{cases}
$$

where we assume the introduced notation (3.6).

Proof. If $\gamma$ is an extremal for $J$, then

$$
\left.\frac{d}{d u}\right|_{u=0} J\left(\alpha_{u}\right)=0, \quad \text { for all variations } \alpha .
$$

Let us choose a variation $\alpha$ of $\gamma$ with variational vector field $W$ given by

$$
W(t)=F(t) \frac{D^{2} \gamma}{d t^{2}}
$$

where $F(t)>0$, for $t \in\left(t_{i}, t_{i+1}\right)$ and $i=0, \ldots, N-1$, and $F\left(t_{i}\right)=0$, for $i=0, \ldots, N$. Then

$$
\left.\frac{d}{d u}\right|_{u=0} J\left(\alpha_{u}\right)=-\lambda \int_{0}^{1} F(t)\left\|\frac{D^{2} \gamma}{d t^{2}}\right\|^{2} d t
$$

and this expression vanishes if and only if, for $t \in\left[t_{i}, t_{i+1}\right]$ and $i=0, \ldots, N-1$,

$$
\frac{D^{2} \gamma}{d t^{2}}=0
$$

In order to prove that $\gamma$ satisfies the boundary conditions (3.11), let us assume that $\gamma$ is an extremal for the functional $J$. Then, as we have just proved, $\gamma$ satisfies (3.10) and the last term in the right-hand side of (3.5) vanishes identically. Therefore, if we consider a variation $\alpha$ of $\gamma$ such that its variational vector field $W$ satisfies

$$
W\left(t_{i}\right)=\lambda\left[\frac{d \gamma}{d t}\left(t_{i}^{+}\right)-\frac{d \gamma}{d t}\left(t_{i}^{-}\right)\right]+\exp _{\gamma\left(t_{i}\right)}^{-1}\left(q_{i}\right), \quad i=0, \ldots, N,
$$

it follows from (3.5) that

$$
\frac{d \gamma}{d t}\left(t_{i}^{+}\right)-\frac{d \gamma}{d t}\left(t_{i}^{-}\right)=-\frac{1}{\lambda} \exp _{\gamma\left(t_{i}\right)}^{-1}\left(q_{i}\right)
$$

which proves the theorem.

Two immediate consequences of Theorem 3.2 are given below.

Proposition 3.1. (i) If, in conditions (3.10)-(3.11) of Theorem 3.2, we consider $\lambda$ converging to 0 , then we obtain a broken geodesic on $M$ interpolating the points $q_{0}, \ldots, q_{N}$ at the instants of time $t_{0}, \ldots, t_{N}$, respectively.

(ii) Under the same conditions, if we consider $\lambda$ converging to $+\infty$, then we obtain a single point in $M$. 
From Theorem 3.2, we can give explicit solutions for (3.10)-(3.11) as long as explicit forms for geodesics on Riemannian manifolds are available. Such is the case for Euclidean spaces, connected and compact Lie groups, and spheres. We will analyze these three cases separately and prove that in conditions of Proposition 3.1(ii), we obtain the Riemannian mean of points $q_{0}, \ldots, q_{N}$, presented in Section 2 .

\subsection{The particular case when $M=\mathbb{R}^{n}$}

When the Riemannian manifold $M$ is the Euclidean space $\mathbb{R}^{n}$ endowed with the usual inner product,

$$
\langle x, y\rangle=x^{\top} y, \quad x, y \in \mathbb{R}^{n},
$$

the counterpart of the functional $J$ defined in Section 3 by (3.3) is

$$
J(\gamma)=\frac{1}{2} \sum_{i=0}^{N}\left\langle q_{i}-\gamma\left(t_{i}\right), q_{i}-\gamma\left(t_{i}\right)\right\rangle+\frac{\lambda}{2} \int_{0}^{1}\left\langle\frac{d \gamma}{d t}, \frac{d \gamma}{d t}\right\rangle d t
$$

and the counterpart of Theorem 3.2 is stated next.

THEOREM 3.3. A necessary condition for $\gamma$ to be an extremal for the functional $J$, given by (3.12), is that for $t \in\left[t_{i}, t_{i+1}\right]$ and $i=0, \ldots, N-1, \gamma$ satisfies

$$
\frac{d^{2} \gamma}{d t^{2}}=0
$$

and at the knot points $t_{i}$, it satisfies

$$
\frac{d^{j} \gamma}{d t^{j}}\left(t_{i}^{+}\right)-\frac{d^{j} \gamma}{d t^{j}}\left(t_{i}^{-}\right)= \begin{cases}0, & j=0(i=1, \ldots, N-1), \\ -\frac{1}{\lambda}\left(q_{i}-\gamma\left(t_{i}\right)\right), & j=1(i=0, \ldots, N),\end{cases}
$$

where we assume that

$$
\frac{d \gamma}{d t}\left(0^{-}\right)=\frac{d \gamma}{d t}\left(1^{+}\right)=0
$$

We will prove that conditions (3.13)-(3.14) are also sufficient for the achievement of the minimum value for $J$.

THEOREM 3.4. For each $\lambda \in \mathbb{R}^{+}$, there exists a unique solution of (3.13)-(3.14) which is the minimizer for the functional $J$ given by (3.12).

Proof. Equation (3.13) can be integrated explicitly in each interval $\left[t_{i}, t_{i+1}\right]$, and we can write

$$
\gamma(t)= \begin{cases}a_{i}+b_{i}\left(t-t_{i}\right), & t_{i} \leqslant t<t_{i+1}(i=0, \ldots, N-1), \\ a_{N}, & t=t_{N},\end{cases}
$$

where the coefficients $a_{i}, b_{i} \in \mathbb{R}^{n}$.

Introducing the positive quantities

$$
h_{i}=t_{i+1}-t_{i}
$$

for $i=0, \ldots, N-1$, for $k=0$ in the system of equations (3.14) we obtain

$$
\gamma\left(t_{i+1}^{+}\right)=\gamma\left(t_{i+1}^{-}\right) \quad \Longrightarrow \quad a_{i+1}-a_{i}=h_{i} b_{i},
$$

for $i=0, \ldots, N-1$, since $\gamma\left(t_{N}^{-}\right)=a_{N}$. 
Now, for $k=1$ in the system of equations (3.14) we obtain

$$
\begin{gathered}
\frac{d \gamma}{d t}\left(t_{0}^{+}\right)=-\frac{1}{\lambda}\left(q_{0}-a_{0}\right) \Longrightarrow b_{0}=-\frac{1}{\lambda}\left(q_{0}-a_{0}\right), \\
\frac{d \gamma}{d t}\left(t_{i}^{+}\right)-\frac{d \gamma}{d t}\left(t_{i}^{-}\right)=-\frac{1}{\lambda}\left(q_{i}-a_{i}\right) \Longrightarrow b_{i}-b_{i-1}=-\frac{1}{\lambda}\left(q_{i}-a_{i}\right) \quad(i=1, \ldots, N-1), \\
-\frac{d \gamma}{d t}\left(t_{N}^{-}\right)=-\frac{1}{\lambda}\left(q_{N}-a_{N}\right) \Longrightarrow-b_{N-1}=-\frac{1}{\lambda}\left(q_{N}-a_{N}\right) .
\end{gathered}
$$

Introducing the matrices

$$
\begin{aligned}
& L=\left[\begin{array}{ccccc}
1 & 0 & 0 & \cdots & 0 \\
-1 & 1 & 0 & \cdots & 0 \\
\vdots & & & \cdots & \vdots \\
0 & \cdots & 0 & -1 & 1 \\
0 & \cdots & 0 & 0 & -1
\end{array}\right] \in \mathbb{R}^{(N+1) \times N} \\
& D=\left[\begin{array}{cccc}
h_{0} & & \cdots & 0 \\
0 & h_{1} & \cdots & 0 \\
\vdots & & \ddots & \vdots \\
0 & \cdots & 0 & h_{N-1}
\end{array}\right] \in \mathbb{R}^{N \times N} \\
& P=\left[\begin{array}{c}
q_{0}^{\top} \\
q_{1}^{\top} \\
\vdots \\
q_{N}^{\top}
\end{array}\right]=\left[\begin{array}{cccc}
q_{0}^{1} & q_{0}^{2} & \cdots & q_{0}^{n} \\
q_{1}^{1} & q_{1}^{2} & \cdots & q_{1}^{n} \\
\vdots & \vdots & \vdots & \vdots \\
q_{N}^{1} & q_{N}^{2} & \cdots & q_{N}^{n}
\end{array}\right] \in \mathbb{R}^{(N+1) \times n}
\end{aligned}
$$

and, analogously,$$
A=\left[\begin{array}{c}
a_{0}^{\top} \\
a_{1}^{\top} \\
\vdots \\
a_{N}^{\top}
\end{array}\right] \in \mathbb{R}^{(N+1) \times n} \quad \text { and } \quad B=\left[\begin{array}{c}
b_{0}^{\top} \\
b_{1}^{\top} \\
\vdots \\
b_{N-1}^{\top}
\end{array}\right] \in \mathbb{R}^{N \times n}
$$

the system of equations (3.16)-(3.17) can be written as the system of matrix equations

$$
\left\{\begin{array}{l}
-L^{\top} A=D B, \\
L B=-\frac{1}{\lambda}(P-A) .
\end{array}\right.
$$

Now, given the fact that $L$ has full rank and $D$ is a positive definite matrix, the above system of matrix equations is uniquely determined, and its solution is given by

$$
\left\{\begin{array}{l}
B=-\left(\lambda L^{\top} L+D\right)^{-1} L^{\top} P, \\
A=P-L\left(L^{\top} L+\frac{D}{\lambda}\right)^{-1} L^{\top} P .
\end{array}\right.
$$

Therefore, there is a unique solution for (3.13)-(3.14), and this solution is, in fact, the minimizer for $J$.

We already know from Proposition 3.1 that when $\lambda$ goes to $+\infty$, the solution of (3.13)(3.14) converges to a point in $\mathbb{R}^{n}$. Our next result states that the latter is the center of mass of the points $q_{0}, \ldots, q_{N}$. 
THEOREM 3.5. When $\lambda$ goes to $+\infty$, the solution of (3.13)-(3.14) converges to the center of mass of the points $q_{0}, \ldots, q_{N}$.

Proof. As we have shown in Theorem 3.4, integrating conditions (3.13)-(3.14) is equivalent to determining the coefficients $a_{i}$ and $b_{i}$ satisfying equations (3.16)-(3.17).

However, we can eliminate the coefficients $b_{i}$ in the system of equations (3.17) by noting that the system

$$
\left\{\begin{array}{l}
b_{0}=-\frac{1}{\lambda}\left(q_{0}-a_{0}\right) \\
b_{1}=-\frac{1}{\lambda}\left(q_{0}-a_{0}\right)-\frac{1}{\lambda}\left(q_{1}-a_{1}\right) \\
\vdots \\
b_{N-1}=-\frac{1}{\lambda}\left(q_{0}-a_{0}\right)-\frac{1}{\lambda}\left(q_{1}-a_{1}\right)-\cdots-\frac{1}{\lambda}\left(q_{N-1}-a_{N-1}\right), \\
-b_{N-1}=-\frac{1}{\lambda}\left(q_{N}-a_{N}\right)
\end{array}\right.
$$

is equivalent to

$$
\left\{\begin{array}{l}
b_{0}=-\frac{1}{\lambda}\left(q_{0}-a_{0}\right), \\
b_{1}=-\frac{1}{\lambda}\left(q_{0}-a_{0}\right)-\frac{1}{\lambda}\left(q_{1}-a_{1}\right), \\
\vdots \\
b_{N-1}=-\frac{1}{\lambda}\left(q_{0}-a_{0}\right)-\frac{1}{\lambda}\left(q_{1}-a_{1}\right)-\cdots-\frac{1}{\lambda}\left(q_{N-1}-a_{N-1}\right), \\
\sum_{i=0}^{N}\left(q_{i}-a_{i}\right)=0
\end{array}\right.
$$

and therefore, when we consider $\lambda \rightarrow+\infty$ in the above system of equations, we obtain

$$
\left\{\begin{array}{l}
b_{0}=0 \\
b_{1}=0 \\
\vdots \\
b_{N-1}=0 \\
\sum_{i=0}^{N}\left(q_{i}-a_{i}\right)=0
\end{array}\right.
$$

But now, since each $b_{i}=0$, for $i=0, \ldots, N-1$, from the system of equations (3.16) we conclude that $a_{i+1}=a_{i}$, for $i=0, \ldots, N-1$ and therefore

$$
a_{i}=a_{0}, \quad i=0, \ldots, N,
$$

which, according to the last equation in (3.20), means that the solution of equations (3.13)(3.14) when $\lambda \rightarrow+\infty$, is given by

$$
\gamma(t)=\frac{1}{N+1} \sum_{i=0}^{N} q_{i} .
$$

The next result is an immediate consequence of the last two theorems.

COROLlary 3.1. With the notation introduced previously, the matrix A given by

$$
A=P-L\left(L^{\top} L\right)^{-1} L^{\top} P
$$

has all the row vectors equal to the vector components of the center of mass of the points $q_{0}, \ldots, q_{N}$, given by (3.21). 

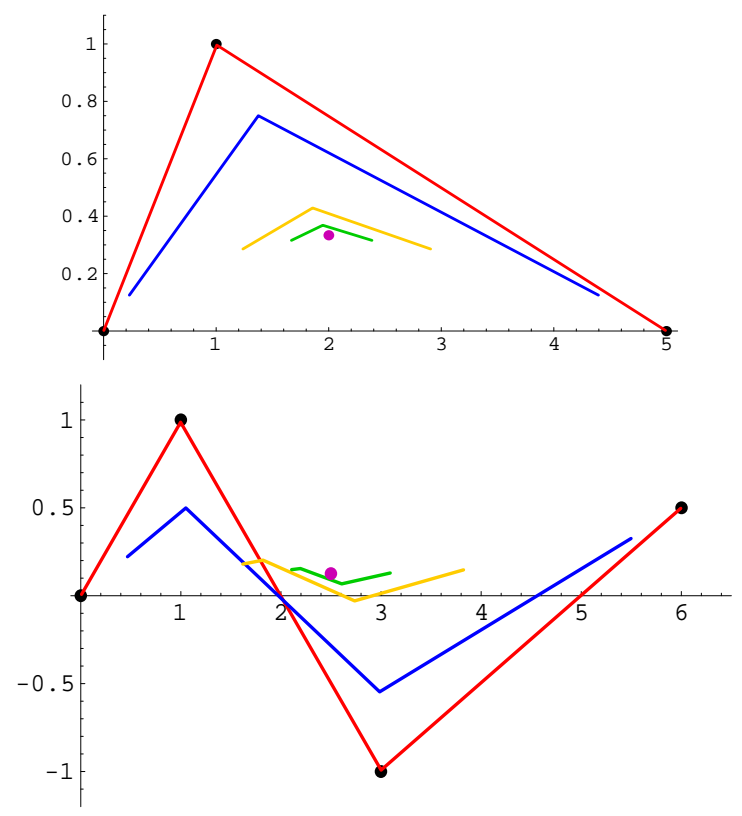

Figure 1: Broken geodesics in the Euclidean space $\mathbb{R}^{2}$ that converge to the center of mass when $\lambda \rightarrow+\infty$.

We finish this subsection by referring to Figure 1, which illustrates the results introduced above for the case when we are given three and four points in the Euclidean space $\mathbb{R}^{2}$.

\subsection{The particular case of connected and compact Lie groups}

In this section we will analyze our variational problem in the case of a connected and compact Lie group $G$. We retain the terminology introduced in Section 2.

We begin with the counterpart of Theorem 3.2 to this case.

THEOREM 3.6. A necessary condition for $\gamma$ to be an extremal for the functional $J$, given by (3.3), is that for $t \in\left[t_{i}, t_{i+1}\right]$ and $i=0, \ldots, N-1, \gamma$ satisfies

$$
\frac{D^{2} \gamma}{d t^{2}}=0
$$

and at the knot points $t_{i}$, it satisfies

$$
\frac{d^{j} \gamma}{d t^{j}}\left(t_{i}^{+}\right)-\frac{d^{j} \gamma}{d t^{j}}\left(t_{i}^{-}\right)= \begin{cases}0, & j=0(i=1, \ldots, N-1), \\ -\frac{1}{\lambda} \gamma\left(t_{i}\right) \log \left(\gamma\left(t_{i}\right)^{-1} q_{i}\right), & j=1(i=0, \ldots, N),\end{cases}
$$

where we assume that

$$
\frac{d \gamma}{d t}\left(0^{-}\right)=\frac{d \gamma}{d t}\left(1^{+}\right)=0
$$


Proof. For the proof, it is only necessary to see that we can explicitly parameterize the geodesic joining $\gamma\left(t_{i}\right)$ (at $s=0$ ) to $q_{i}$ (at $s=1$ ), (assuming that $q_{i}$ and $\gamma\left(t_{i}\right)$ are sufficiently close enough) as

$$
\begin{aligned}
\beta:[0,1] & \longrightarrow G \\
s & \longmapsto \beta(s)=\gamma\left(t_{i}\right) \exp \left(s \log \left(\gamma\left(t_{i}\right)^{-1} q_{i}\right)\right)
\end{aligned}
$$

and that the velocity vector of $\beta$ at the point $\gamma\left(t_{i}\right)$ is $\gamma\left(t_{i}\right) \log \left(\gamma\left(t_{i}\right)^{-1} q_{i}\right)$.

Now we will prove the counterpart of Theorem 3.5 on Euclidean spaces for this case.

THEOREM 3.7. When $\lambda$ goes to $+\infty$, the solution of conditions (3.22)-(3.23) converges to the Riemannian mean of the points $q_{0}, \ldots, q_{N}$.

Proof. Since geodesics in $G$, associated to the bi-invariant Riemannian metric on $G$, are the one-parameter subgroups or their translates, we can explicitly integrate equation (3.22) in each interval $\left[t_{i}, t_{i+1}\right]$, for $i=0, \ldots, N-1$, and write

$$
\gamma(t)= \begin{cases}p_{i} \exp \left(\left(t-t_{i}\right) X_{i}\right), & t_{i} \leqslant t<t_{i+1}(i=0, \ldots, N-1), \\ p_{N}, & t=t_{N} .\end{cases}
$$

If we introduce $h_{i}=t_{i+1}-t_{i}$, for $i=0, \ldots, N-1$, we obtain, for $k=0$ in the system of equations (3.23),

$$
\gamma\left(t_{i+1}^{+}\right)=\gamma\left(t_{i+1}^{-}\right) \quad \Longrightarrow \quad X_{i}=\frac{1}{h_{i}} \log \left(p_{i}^{-1} p_{i+1}\right),
$$

for $i=0, \ldots, N-1$.

Now, for $k=1$ in the system of equations (3.23), we obtain

$$
\left\{\begin{array}{l}
p_{0} X_{0}=-\frac{1}{\lambda} p_{0} \log \left(p_{0}^{-1} q_{0}\right) \\
p_{1} X_{1}-p_{0} X_{0} \exp \left(h_{0} X_{0}\right)=-\frac{1}{\lambda} p_{1} \log \left(p_{1}^{-1} q_{1}\right) \\
\vdots \\
p_{N-1} X_{N-1}-p_{N-2} X_{N-2} \exp \left(h_{N-2} X_{N-2}\right)=-\frac{1}{\lambda} p_{N-1} \log \left(p_{N-1}^{-1} q_{N-1}\right) \\
-p_{N-1} X_{N-1} \exp \left(h_{N-1} X_{N-1}\right)=-\frac{1}{\lambda} p_{N} \log \left(p_{N}^{-1} q_{N}\right)
\end{array}\right.
$$

which is equivalent to

$$
\left\{\begin{array}{l}
p_{0} X_{0}=-\frac{1}{\lambda} p_{0} \log \left(p_{0}^{-1} q_{0}\right), \\
p_{1} X_{1}=-\frac{1}{\lambda} p_{0} \log \left(p_{0}^{-1} q_{0}\right) \exp \left(h_{0} X_{0}\right)-\frac{1}{\lambda} p_{1} \log \left(p_{1}^{-1} q_{1}\right), \\
\vdots \\
p_{N-1} X_{N-1}=-\frac{1}{\lambda} \sum_{i=0}^{N-2} p_{i} \log \left(p_{i}^{-1} q_{i}\right) \prod_{j=i}^{N-2} \exp \left(h_{j} X_{j}\right)-\frac{1}{\lambda} p_{N-1} \log \left(p_{N-1}^{-1} q_{N-1}\right), \\
-p_{N-1} X_{N-1} \exp \left(h_{N-1} X_{N-1}\right)=-\frac{1}{\lambda} p_{N} \log \left(p_{N}^{-1} q_{N}\right),
\end{array}\right.
$$


which is still equivalent to

$$
\left\{\begin{array}{l}
p_{0} X_{0}=-\frac{1}{\lambda} p_{0} \log \left(p_{0}^{-1} q_{0}\right), \\
p_{1} X_{1}=-\frac{1}{\lambda} p_{0} \log \left(p_{0}^{-1} q_{0}\right) \exp \left(h_{0} X_{0}\right)-\frac{1}{\lambda} p_{1} \log \left(p_{1}^{-1} q_{1}\right), \\
\vdots \\
p_{N-1} X_{N-1}=-\frac{1}{\lambda} \sum_{i=0}^{N-2} p_{i} \log \left(p_{i}^{-1} q_{i}\right) \prod_{j=i}^{N-2} \exp \left(h_{j} X_{j}\right)-\frac{1}{\lambda} p_{N-1} \log \left(p_{N-1}^{-1} q_{N-1}\right), \\
\sum_{i=0}^{N-1} p_{i} \log \left(p_{i}^{-1} q_{i}\right) \prod_{j=i}^{N-1} \exp \left(h_{j} X_{j}\right)+p_{N} \log \left(p_{N}^{-1} q_{N}\right)=0 .
\end{array}\right.
$$

When we consider $\lambda$ going to $+\infty$ in the above system of equations, we get

$$
\left\{\begin{array}{l}
X_{0}=0 \\
X_{1}=0 \\
\vdots \\
X_{N-1}=0 \\
\sum_{i=0}^{N} p_{i} \log \left(p_{i}^{-1} q_{i}\right)=0
\end{array}\right.
$$

and according to equations (3.24), we obtain

$$
\log \left(p_{i}^{-1} p_{i+1}\right)=0
$$

for $i=0, \ldots, N-1$, which implies that

$$
p_{i+1}=p_{i}
$$

for $i=0, \ldots, N-1$.

Thus the solution of equations (3.22)-(3.23), when $\lambda$ goes to $+\infty$, namely

$$
\gamma(t)=p_{0},
$$

satisfies the equation

$$
\sum_{i=0}^{N} \log \left(p_{0}^{-1} q_{i}\right)=0,
$$

which characterizes the Riemannian mean of the points $q_{0}, \ldots, q_{N}$, given in Proposition 2.1.

\subsection{The particular case of spheres}

We start by stating the counterpart of Theorem 3.2 in the $n$-dimensional unit sphere $S^{n}$.

THEOREM 3.8. A necessary condition for $\gamma$ to be an extremal for the functional $J$, given by (3.3), is that for $t \in\left[t_{i}, t_{i+1}\right]$ and $i=0, \ldots, N-1, \gamma$ satisfies

$$
\frac{D^{2} \gamma}{d t^{2}}=0
$$


and at the knot points $t_{i}$, it satisfies,

$$
\frac{d^{j} \gamma}{d t^{j}}\left(t_{i}^{+}\right)-\frac{d^{j} \gamma}{d t^{j}}\left(t_{i}^{-}\right)= \begin{cases}0, & j=0(i=1, \ldots, N-1), \\ -\frac{1}{\lambda} \frac{\alpha_{i}}{\sin \alpha_{i}}\left(q_{i}-\gamma\left(t_{i}\right) \cos \alpha_{i}\right), & j=1(i=0, \ldots, N),\end{cases}
$$

where $\alpha_{i}=\cos ^{-1}\left\langle\gamma\left(t_{i}\right), q_{i}\right\rangle$, for $i=0, \ldots, N$, and we assume that

$$
\frac{d \gamma}{d t}\left(0^{-}\right)=\frac{d \gamma}{d t}\left(1^{+}\right)=0
$$

Proof. To prove the theorem, it is enough to check that the velocity vector, at the point $\gamma\left(t_{i}\right)$, of the shortest geodesic arc joining $\gamma\left(t_{i}\right)$ (at $\left.t=0\right)$ to $q_{i}$ (at $t=1$ ) is given by

$$
\frac{\alpha_{i}}{\sin \alpha_{i}}\left(q_{i}-\gamma\left(t_{i}\right) \cos \alpha_{i}\right)
$$

where $\alpha_{i}=\cos ^{-1}\left\langle\gamma\left(t_{i}\right), q_{i}\right\rangle$.

Now we will prove the counterparts of Theorems 3.5 and 3.7 for the unit $n$-sphere $S^{n}$.

THEOREM 3.9. When $\lambda$ goes to $+\infty$, the solution of conditions (3.25)-(3.26) converges to the Riemannian mean of the points $q_{0}, \ldots, q_{N}$.

Proof. Proceeding analogously to what has been done in the proof of Theorem 3.7, we explicitly integrate equation (3.25) in each interval $\left[t_{i}, t_{i+1}\right]$, for $i=0, \ldots, N-1$, according to the explicit form for parameterized geodesics on $S^{n}$ given by (2.5), and we consider

$$
\begin{aligned}
& \gamma(t)= \\
& \begin{cases}p_{i} \cos \left(\left\|v_{i}\right\|\left(t-t_{i}\right)\right)+\frac{v_{i}}{\left\|v_{i}\right\|} \sin \left(\left\|v_{i}\right\|\left(t-t_{i}\right)\right), & t_{i} \leqslant t<t_{i+1}(i=0, \ldots, N-1), \\
p_{N}, & t=t_{N} .\end{cases}
\end{aligned}
$$

For $k=0$ in the system of equations (3.26), we obtain

$$
\gamma\left(t_{i+1}^{+}\right)=\gamma\left(t_{i+1}^{-}\right) \quad \Longrightarrow \quad p_{i+1}=p_{i} \cos \left(h_{i}\left\|v_{i}\right\|\right)+\frac{v_{i}}{\left\|v_{i}\right\|} \sin \left(h_{i}\left\|v_{i}\right\|\right),
$$

for $i=0, \ldots, N-1$.

Now, since for $t \in\left[t_{i}, t_{i+1}[\right.$, we have

$$
\frac{d \gamma}{d t}(t)=-p_{i}\left\|v_{i}\right\| \sin \left(\left\|v_{i}\right\|\left(t-t_{i}\right)\right)+v_{i} \cos \left(\left\|v_{i}\right\|\left(t-t_{i}\right)\right)
$$

for $k=1$ in the system of equations (3.26), we get

$$
\left\{\begin{aligned}
v_{0}=-\frac{1}{\lambda} \frac{\alpha_{0}}{\sin \alpha_{0}}\left(q_{0}-p_{0} \cos \alpha_{0}\right), & \\
v_{1}+p_{0}\left\|v_{0}\right\| \sin \left(h_{0}\left\|v_{0}\right\|\right)-v_{0} \cos \left(\left\|v_{0}\right\| h_{0}\right) & =-\frac{1}{\lambda} \frac{\alpha_{1}}{\sin \alpha_{1}}\left(q_{1}-p_{1} \cos \alpha_{1}\right), \\
v_{N-1}+p_{N-2}\left\|v_{N-2}\right\| \sin \left(h_{N-2}\left\|v_{N-2}\right\|\right)- & v_{N-2} \cos \left(\left\|v_{N-2}\right\| h_{N-2}\right), \\
& =-\frac{1}{\lambda} \frac{\alpha_{N-1}}{\sin \alpha_{N-1}}\left(q_{N-1}-p_{N-1} \cos \alpha_{N-1}\right), \\
p_{N-1}\left\|v_{N-1}\right\| \sin \left(\left\|v_{N-1}\right\| h_{N-1}\right)-v_{N-1} \cos & \left(\left\|v_{N-1}\right\| h_{N-1}\right) \\
& =-\frac{1}{\lambda} \frac{\alpha_{N}}{\sin \alpha_{N}}\left(q_{N}-p_{N} \cos \alpha_{N}\right),
\end{aligned}\right.
$$


which is equivalent to

$$
\left\{\begin{aligned}
v_{0}= & -\frac{1}{\lambda} \frac{\alpha_{0}}{\sin \alpha_{0}}\left(q_{0}-p_{0} \cos \alpha_{0}\right), \\
v_{1}= & -p_{0}\left\|v_{0}\right\| \sin \left(h_{0}\left\|v_{0}\right\|\right)-\frac{\alpha_{0}}{\lambda \sin \alpha_{0}}\left(q_{0}-p_{0} \cos \alpha_{0}\right) \cos \left(\left\|v_{0}\right\| h_{0}\right) \\
& -\frac{\alpha_{1}}{\lambda \sin \alpha_{1}}\left(q_{1}-p_{1} \cos \alpha_{1}\right) \\
\vdots & \\
v_{N-1}= & -p_{N-2}\left\|v_{N-2}\right\| \sin \left(h_{N-2}\left\|v_{N-2}\right\|\right)-\sum_{i=0}^{N-3} p_{i}\left\|v_{i}\right\| \sin \left(h_{i}\left\|v_{i}\right\|\right) \prod_{j=i+1}^{N-2} \cos \left(\left\|v_{j}\right\| h_{j}\right) \\
& -\frac{1}{\lambda} \sum_{i=0}^{N-2} \frac{\alpha_{i}}{\sin \alpha_{i}}\left(q_{i}-p_{i} \cos \alpha_{i}\right) \prod_{j=i}^{N-2} \cos \left(h_{j}\left\|v_{j}\right\|\right) \\
& \quad \frac{\alpha_{N-1}}{\lambda \sin \alpha_{N-1}}\left(q_{N-1}-p_{N-1} \cos \alpha_{N-1}\right) \\
p_{N-1}\left\|\lambda v_{N-1}\right\| \sin \left(\left\|v_{N-1}\right\| h_{N-1}\right)+\sum_{i=0}^{N-2} p_{i}\left\|\lambda v_{i}\right\| \sin \left(h_{i}\left\|v_{i}\right\|\right) \prod_{j=i+1}^{N-1} \cos \left(\left\|v_{j}\right\| h_{j}\right) & \\
& +\sum_{i=0}^{N-1} \frac{\alpha_{i}}{\sin \alpha_{i}}\left(q_{i}-p_{i} \cos \alpha_{i}\right) \prod_{j=i}^{N-1} \cos \left(h_{j}\left\|v_{j}\right\|\right)+\frac{\alpha_{N}}{\sin \alpha_{N}}\left(q_{N}-p_{N} \cos \alpha_{N}\right) \\
= & 0 .
\end{aligned}\right.
$$

Therefore, when $\lambda$ goes to $+\infty$ in the above system of equations, we obtain

$$
\left\{\begin{array}{l}
v_{0}=0, \\
v_{1}=0, \\
\vdots \\
v_{N-1}=0, \\
\sum_{i=0}^{N} \frac{\alpha_{i}}{\sin \alpha_{i}}\left(q_{i}-p_{i} \cos \alpha_{i}\right)=0 .
\end{array}\right.
$$

Now, since when $\lambda$ goes to $+\infty, v_{i}=0$, for $i=0, \ldots, N-1$, from equations (3.27) we conclude that

$$
p_{i+1}=p_{i}
$$

for $i=0, \ldots, N-1$, which means that the solution of (3.25)-(3.26), namely

$$
\gamma(t)=p_{0}, \quad \forall t \in[0,1],
$$

satisfies the equation

$$
\sum_{i=0}^{N} \frac{\alpha_{i}}{\sin \alpha_{i}}\left(q_{i}-p_{0} \cos \alpha_{i}\right)=0
$$

The proof is now complete if one compares this equation with the equation for the Riemannian mean of the points $q_{0}, \ldots, q_{N}$, on the sphere $S^{n}$, given in Proposition 2.2. 
Acknowledgements. The first two authors were supported in part by the Calouste Gulbenkian Foundation and the Institute of Systems and Robotics - Coimbra. Support for the first author was also provided by the PRODEP 5.3 program (UE-FSE).

The work was initiated while the authors were visiting each other in Portugal and Australia in 2004 and 2005.

National ICT Australia is funded by the Australian Government's Department of Communications, Information Technology and the Arts and the Australian Research Council through Backing Australia's Ability and the ICT Centre of Excellence Program.

\section{References}

1. Roy L. Adler, Jean-Pierre Dedieu, Joseph Y. Margulies, Marco Martens and Mike Shub, 'Newton's method on Riemannian manifolds and a geometric model for the human spine', IMA J. Numer. Anal. 22 (2002) 359-390. 87

2. S. R. Buss and J. P. FILlmore, 'Spherical averages and applications to spherical splines and interpolation', ACM Trans. Graphics 2 (2001) 95-126. 86, 90

3. M. P. DO CARMO, Riemannian geometry (Birkhäuser, Boston, 1992). 92

4. G. A. Galperin, 'A concept of mass center of a system of material points in the constant curvature spaces', Comm. Math. Phys. 154 (1993) 63-84. 86

5. S. Helgason, Differential geometry, Lie groups and symmetric spaces (Academic Press, Boston, 1978). 88

6. P. E. Jupp and J. T. Kent, 'Fitting smooth paths to spherical data', Appl. Statist. 36 (1987) 34-46. 87

7. H. KARCHER, 'Riemannian center of mass and mollifier smoothing', Comm. Pure Appl. Math. 30 (1977) 509-541. 86, 87, 88

8. S. KobAyashi and K. Nomizu, Foundations of differential geometry, Interscience Tracts in Pure and Applied Mathematics 2 (Interscience Publishers, New York, 1969). 86

9. A. K. Krakowski, 'Geometrical methods of inference', $\mathrm{PhD}$ Thesis, Department of Mathematics and Statistics, University of Western Australia, Australia, 2002. 86, 87, $88,89,92$

10. M. Lazard and J. Tits, 'Domaines d'injectivité de l'application exponentielle', Topology 4 (1966) 315-322. 89

11. L. Machado and F. Silva Leite, 'Fitting smooth paths on Riemannian manifolds', preprint no. 04-31, Department of Mathematics, University of Coimbra, 2004. 87

12. J. W. Milnor, Morse theory (Princeton University Press, Princeton, New Jersey, 1963). $87,88,92$

13. M. MoAKHer, 'Means and averaging in the group of rotations', SIAM. J. Matrix Anal. Appl. 24 (2001) 1-16. 86, 87

14. M. MoAKHer, 'A differential geometric approach to the arithmetic and geometric means of operators in some symmetric spaces', SIAM. J. Matrix Anal. Appl. 26 (2005) 735-747. 86, 87

15. H. Munthe-KaAs, R. G. W. Quispel and A. Zanna, 'The polar decomposition on Lie groups with involutive automorphisms', Found. Comp. Math. 1:3 (2001) 297-324. 89 
Luís Machado lmigueleutad.pt

http://home.utad.pt/ lmiguel

Department of Mathematics

University of Trás-os-Montes e Alto Douro

5000-911 Vila Real

Portugal

F. Silva Leite fleite@mat.uc.pt

http://www.mat.uc.pt/ fleite

Department of Mathematics and Institute of Systems and Robotics

University of Coimbra

3001-454 Coimbra

Portugal

Knut Hüper knut.hueper@nicta.com.au

http://www.rsise.anu.edu.au/ hueper

National ICT Australia

Canberra Research Laboratory

SEACS Program

Locked Bag 8001

Canberra ACT 2601

Australia

and

Department of Information Engineering

Research School of Information Sciences and Engineering

The Australian National University

Canberra ACT 0200

Australia 